\title{
Development of a quantitative loop-mediated isothermal amplification assay for the field detection of Erysiphe necator
}



Plant pathogen detection systems have been useful tools to monitor inoculum presence and initiate management schedules. More recently, a LAMP assay was successfully designed for field use in the grape powdery mildew pathosystem; however, false negatives or false positives were prevalent in grower-conducted assays due to the difficulty in perceiving the magnesium pyrophosphate precipitate at low DNA concentrations. A quantitative LAMP (qLAMP) assay using a FRET-based probe was assessed by grape growers in the Willamette Valley of Oregon. Custom impaction spore samplers were placed at a research vineyard and 6 commercial vineyard locations, and were tested bi-weekly by the lab and by growers. Grower conducted qLAMP assays used a beta-version of the Smart-DART handheld LAMP reaction devices (Diagenetix Inc., Honolulu, HI), connected to Android 4.4 enabled, Bluetooth-capable Nexus 7 tablets for output. Quantification by a qPCR assay was assumed correct to compare the lab and grower qLAMP assay quantification. Growers were able to conduct and interpret qLAMP results; however, the $E$. necator inoculum quantification was unreliable using the beta-SMART-dart devices. The qLAMP assay developed was sensitive to 1 spore in early testing of the assay, but decreased to $>20$ spores by the end of the trial. The qLAMP assay is not likely a suitable management tool for grape powdery mildew due to losses in sensitivity and decreasing costs and portability for other more reliable molecular tools. 
1

3 L. D. Thiessen*a , T. M. Neill ${ }^{b}$, and W. F. Mahaffee ${ }^{b}$

4 a Oregon State University, Department of Botany and Plant Pathology, Corvallis, OR 97331

\section{Development of a Quantitative Loop-Mediated Isothermal Amplification Assay for the}

\section{Field Detection of Erysiphe necator}

${ }^{\mathrm{b}}$ U.S. Department of Agriculture-Agricultural Research Service, Corvallis, OR 97331

*Corresponding author: L. D. Thiessen

E-mail: 1dthiess@ncsu.edu

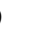

(10)


24 Abstract

25 Plant pathogen detection systems have been useful tools to monitor inoculum presence and initiate management schedules. More recently, a LAMP assay was successfully designed for

27 field use in the grape powdery mildew pathosystem; however, false negatives or false positives were prevalent in grower-conducted assays due to the difficulty in perceiving the magnesium pyrophosphate precipitate at low DNA concentrations. A quantitative LAMP (qLAMP) assay using a FRET-based probe was assessed by grape growers in the Willamette Valley of Oregon. Custom impaction spore samplers were placed at a research vineyard and 6 commercial vineyard locations, and were tested bi-weekly by the lab and by growers. Grower conducted qLAMP assays used a beta-version of the Smart-DART handheld LAMP reaction devices (Diagenetix Inc., Honolulu, HI), connected to Android 4.4 enabled, Bluetooth-capable Nexus 7 tablets for output. Quantification by a qPCR assay was assumed correct to compare the lab and grower qLAMP assay quantification. Growers were able to conduct and interpret qLAMP results; however, the E. necator inoculum quantification was unreliable using the beta-SMART-dart devices. The qLAMP assay developed was sensitive to 1 spore in early testing of the assay, but decreased to $>20$ spores by the end of the trial. The qLAMP assay is not likely a suitable management tool for grape powdery mildew due to losses in sensitivity and decreasing costs and 41 portability for other more reliable molecular tools. 


\section{Introduction}

Molecular techniques, such as PCR, are capable of being used to detect specific pathogens in air samples with high sensitivity and specificity (Carisse et al. 2009a; Carisse et al. 2009c; Falacy et al. 2007; Thiessen et al. 2016; West et al. 2008a). The detection of airborne pathogen inoculum has been improved through the development of quantitative PCR (qPCR) assays that allow for near real-time monitoring of inoculum concentration (Carisse et al. 2009c; Rogers et al. 2009; Temple \& Johnson 2011; Thiessen et al. 2016). Despite the utility of qPCR to monitor pathogens, it is often impractical due to requirements for experienced laboratory staff and expensive equipment to accurately assess pathogen concentration (Notomi et al. 2000; West et al. 2008b).

Loop-mediated isothermal amplification (LAMP) assays could be an inexpensive alternative for detection in the field or at remote facilities. LAMP can use relatively inexpensive and mobile equipment and utilizes the Bst polymerase that has a high tolerance to reaction inhibitors (Kubota et al. 2011), which allows for quick, minimal DNA extraction protocols. These traits make LAMP useful in field detection assays (Harper et al. 2010; Kubota et al. 2008; Temple \& Johnson 2011; Tomlinson et al. 2007; Tomlinson et al. 2010).

LAMP has been developed for monitoring inoculum in numerous plant pathosystems, including grape powdery mildew (Erysiphe necator), fire blight of pear (Erwinia amylovora), and gray mold (Botrytis cinerea) (Temple \& Johnson 2011; Thiessen et al. 2016; Tomlinson et al. 2010). Traditional LAMP assays produce a magnesium pyrophosphate precipitate when DNA is amplified that can be detected with the human eye; however, in low concentrations of target DNA, precipitate may be difficult to observe (Jenkins et al. 2011; Kubota et al. 2011; Thiessen et al. 2016) or require expensive equipment (Temple and Johnson 2011). Several dyes have been 
explored to improve detection including SYBR green (Notomi et al. 2000), hydroxynaphthol blue (Cardoso et al. 2010), and other synthetic dyes (Fischbach et al. 2015), but the dyes have the potential to inhibit LAMP reactions or require the use of spectrophotometers, which increase labor and equipment costs. The use of a fluorescence resonance energy transfer (FRET) based probe, allows for specific detection of LAMP products and target quantification from field samples without inhibiting amplification (Kubota et al. 2011), and several portable fluorescencereading LAMP devices have been made commercially available, such as the Genie (Optigene Ltd., West Sussex, UK) and Bioranger (Diagenetix Inc., Hawaii). Using a fluorescent probe also reduces potential classification error from visual detection of LAMP products, which may improve the accuracy of pathogen detection and allow for quantification.

Grape powdery mildew, caused by Erysiphe necator, causes damages to grape (Vitis vinifera L.) wherever it is produced. This disease requires numerous applications of fungicides, which are either applied on a calendar schedule from bud break (BBCH 08) until véraison (BBCH 83) or based on disease risk models (Carisse et al. 2009b; Gadoury \& Pearson 1990; Thomas et al. 1994). More recently, fungicide applications have been reduced using inoculum detection systems (Thiessen et al. 2017; Thiessen et al. 2016); however, these systems do not provide in-field inoculum concentration for producers. Additionally, the LAMP assay that was successfully designed for field use in the grape powdery mildew pathosystem had numerous false negatives or false positives, which may have been caused by difficulty in perceiving the magnesium pyrophosphate precipitate, reducing the predictive values of the LAMP assay (Thiessen et al. 2016). A timely and cost-effective system that improves detection of E. necator inoculum throughout the growing season is needed to allow growers to accurately time fungicide 
88

89

90

applications early in the growing season and adjust application intervals based on inoculum concentration.

The purpose of this research was to develop a quantitative molecular assay for commercial implementation that could be used by growers or vineyard consultants for the detection and quantification of airborne $E$. necator inoculum. The specific objectives of this project were to 1) develop a real-time, quantitative LAMP assay that was sensitive and specific to E. necator, and 2) test field use of a mobile, quantitative LAMP device by growers.

\section{Materials and Methods}

Sample Rod Preparation. Sample rods were created by cutting Stainless steel 308LSI welding rods (1.1 $\mathrm{mm}$ in diameter) (Weldcote Metals, Kings Mountain, NC) to $36 \mathrm{~mm}$ lengths, then sterilized and prepared according to Thiessen et al. (2016). To produce a standard curve, conidial suspensions were generated by suspending E. necator conidia from Vitis vinifera $\mathrm{cv}$. 'Chardonnay' vines in a $0.05 \%$ Tween 20 (Sigma-Aldrich, St. Louis, MO) and nuclease-free water solution then pipetting the conidial suspension onto rod sets resulting in rods with 100 , 1000 , or 10,000 conidia per sample. Rods with 1 or 10 spores were created by transferring individual spores with eyelash brush. Six independent spore dilution series were used to generate the standard curve for the quantitative assay. Additionally, a set of sample rods containing 500 conidia was also generated using the conidial suspension to act as a positive control for all DNA extractions and molecular reactions. The rods were air dried prior to processing.

Quantitative LAMP Assay. DNA for qLAMP analysis was extracted using a quick extraction method modified from Thiessen et al. (2016). Spore rods were transferred to 2-ml screw-cap tubes containing $200 \mu 1$ of 5\% Chelex 100 (Sigma Aldrich) in molecular grade, 
111 DEPC-treated water. Tubes containing rods were vortexed for 5 seconds then placed in boiling

112 water for 5 minutes. Tubes were removed from boiling water and vortexed another 5 seconds.

113 The tubes were boiled for another 5 minutes, and then removed and allowed to cool at room

114 temperature for 2 minutes. Samples were centrifuged at 16,000 \& $\mathrm{g}$ for 2 minutes to collect the

115 contents in the tube. Rods were aseptically removed in a laminar flow hood prior to the pellet

116 being processed using the Chelex DNA extraction process (described below). After DNA were

117 extracted and amplified, samples were stored at $-20^{\circ} \mathrm{C}$ for further analyses.

The qLAMP reaction is a modified assay from Thiessen et al. (2016) and Kubota et al.

119 (2011), which was optimized to generate a quantification standard curve (described above). A

120

121

122

123

124

125

126

127

FRET-based probe was designed using the forward loop primer region with a FAM reporter (6carboxyfluorescein) and a quencher strand (Kubota et al. 2011). Each reaction contained $14.8 \mu 1$ of Isothermal Master Mix with no dye (OptiGene Ltd, West Sussex, UK), internal primers FIP EN and BIP EN $(2.4 \mu \mathrm{M})$, external primers F3 EN and B3 EN $(0.24 \mu \mathrm{M})$, forward loop primer FAM strand (FL-F, $0.08 \mu \mathrm{M}$, FAM-ACGC TGAG GACC CGGA TGCG AATG CGGA TGCG GATG CCGA AAAC TGCG ACGA GCCC C), and Quencher strand (Q-strand, $0.08 \mu \mathrm{M}$, TCGG CATC CGCA TCCG CATT CGCA TCCG GGTC CTCA GCGT-BHQ) to create a $25 \mu 1$ reaction (Table 1). Lab-conducted qLAMP (L-qLAMP) reactions were carried out on an ABI StepOne Plus qPCR machine (Applied Biosystems, Grand Island, NY, USA). Reaction conditions were $65^{\circ} \mathrm{C}$ for 45 minutes followed by $80^{\circ} \mathrm{C}$ for 5 minutes. All reactions were run in triplicate

The reaction time threshold $\left(\mathrm{R}_{\mathrm{T}}\right)$ values, measured in minutes, of the spore standards were averaged and used to create a log-linear standard curve against which unknown samples were compared (Fig. 1). A log-linear curve is required to describe the assay because LAMP 
134 amplification rate is faster than exponential due to concatenation of amplicon (Mori et al. 2001).

135 A 500-conidia extraction control, 100 and 1,000-conidia positive controls, as well as non-

136 template controls were included in all reaction setups. Unknowns were compared to the standard

137 curve to determine relative spore quantity. Positive control samples were also compared to the

138

139

140 standard curve to determine extraction efficiency and amplification efficiency. Unknown sample $R_{T}$ values were adjusted based on positive control $R_{T}$ values if the positive controls showed poor alignment to the standard curve. To test the L-qLAMP sensitivity to target DNA, ten separate spore concentration series were created and tested for positive amplification.

Grower Quantitative LAMP Assay. Growers were provided with all equipment and supplies to conduct the DNA extraction and the qLAMP reaction protocol described above. DNA extraction and qLAMP assays were conducted in any location growers deemed appropriate (i.e. office space, winery hallway, tractor barn, kitchen table). For the grower-conducted qLAMP assay (G-qLAMP), frozen aliquots of qLAMP master mix were stored in insulated cryoboxes (VWR North America, Radnor, PA) at -20C until reactions were conducted. All reactions were conducted in beta-version Smart-DART handheld LAMP reaction devices (Diagenetix Inc., Honolulu, HI), which connected to Android 4.4 enabled, Bluetooth-capable Nexus 7 tablets for output (Google, Mountain View, CA). All G-qLAMP reactions were conducted in duplicate including 100-conidia positive controls and non-template controls. Reaction conditions followed the protocol described above.

Smart-DART LAMP devices provided amplification curves and the $\mathrm{R}_{\mathrm{T}}$ values associated with amplification curves. Growers were asked to determine if samples were positive, as indicated by the presence of a sigmoidal amplification curve, or negative, no amplification observed, based on the output from the handheld LAMP device. 
Quantitative PCR Assay. The DNA from collected spore sampler rod pairs was extracted using the PowerSoil ${ }^{\circledR}$ DNA extraction kit (Mo Bio Laboratories, Inc. Carlsbad, CA) following the manufacturer's protocols. In each set of DNA extractions, a set of positive control rods containing 500 E. necator conidia was included as an extraction efficiency control. Erysiphe necator primers developed by Falacy et al. (2007) were paired with a TaqMan® probe with a minor groove binder (Thiessen et al. 2016). All qPCR reactions contained 7.5 $\mu 1 \operatorname{PerfeC}_{\mathrm{T}} \mathrm{a}^{\circledR}$ qPCR ToughMix ${ }^{\circledR}$ (Quanta Biosciences, Gaithersburg, MD), $400 \mathrm{nM}$ final concentrations of each E. necator forward and reverse primers and probe, and $1.5 \mu 1$ extracted sample DNA for a $15 \mu l$ total volume. Reactions were carried out using an ABI StepOne Plus qPCR machine (Applied Biosystems, Foster City, CA). All qPCR reactions were performed in triplicate, and each reaction plate contained the 500 conidia extraction control, 100 and 10,000 conidia positive reaction controls, and template-free negative control.

Cycle threshold $\left(\mathrm{C}_{\mathrm{T}}\right)$ analysis was conducted using ABI StepOne ${ }^{\mathrm{TM}}$ software according to protocols by Thiessen et al. (2016). Spore concentrations were determined for field samples by identifying the average $R_{T}$ value for each triplicate reaction, and comparing this value to the standard curve described below. Average $\mathrm{R}_{\mathrm{T}}$ values of positive controls $(100,500$, and 10,000 conidia) from each set of qPCR reactions were used to confirm the efficiency and to the suitability of the standard curve for determining conidia concentration of unknowns. The standard curve was generated by creating five independent, ten-fold conidial dilution series on the stainless-steel sampling rods 1 to $1 \times 10^{5}$ conidia (described above), DNA was extracted using the PowerSoil Kit (described above), and the average $\mathrm{R}_{\mathrm{T}}$ values for each conidia quantity from the five independent DNA extractions was used to fit a linear curve. 
Field Sample Collection and Assay Comparison. Custom impaction spore samplers (Thiessen et al. 2016), were placed at a research vineyard and 6 commercial vineyard locations within the Willamette Valley of Oregon. Each spore sampler contained a pair of sample rods described above. Spore samplers were run continuously, sampling $45 \mathrm{~L} / \mathrm{min}$, and sample rods were replaced daily or every Monday and Thursday (bi-weekly). Three spore samplers were placed at each of the 6 commercial vineyards that were collected by growers bi-weekly. The growers completely maintained one trap, processing all sample rods derived from that trap.

Sample rods from the other two traps were collected by the growers and transported to the lab for processing with the L-qLAMP assay and the qPCR assay. At the Oregon State University Botany and Plant Pathology research vineyard, paired spore samplers, one for the qPCR assay and one for the qLAMP assay, were collected and processed by laboratory personnel on a daily and biweekly schedule.

Spore samplers for the L-qLAMP and the qPCR assays were deployed on April 15, 2013 and April 14, 2014 and sample rods were collected from bud break until véraison (BBCH 83). Spore samplers for the G-qLAMP assay were deployed April 14, 2014 and were collected until July 1, 2014. Estimates of airborne inoculum concentration derived using qPCR and qLAMP were compared to assess the accuracy of the qLAMP procedure. The G-qLAMP assay detection results were compared to the L-qLAMP assay and qPCR detection data as described below.

Data Analysis. Data was analyzed using R 3.2.1. Detections from samples collected and quantified with L-qLAMP assay were compared to qPCR assay detections using a Student's Ttest. The G-qLAMP detection results were compared to L-qLAMP detection results using a $2 \times 2$ contingency table whereby the L-qLAMP results were assumed correct. Both the L-qLAMP and G-qLAMP spore detections were evaluated using a $2 \times 2$ contingency table whereby the qPCR 
202

203

assay results were assumed correct. The qLAMP assay detection accuracy, true positive proportion, true negative proportion, Fisher's Exact test, and Chi-squared test were assessed comparing the qLAMP detection results to the qPCR detection results.

\section{Results}

qLAMP Assay Sensitivity. The qLAMP assay showed high sensitivity to E. necator conidia DNA when 10 separate spore dilution series were tested (Fig. 1) with $80 \%$ of 1 conidia samples amplifying using the qLAMP assay. All other spore quantities tested showed 100\% amplification sensitivity within the qLAMP assay.

qLAMP Quantification. The qLAMP assay standard curve development resulted in a standard curve $\left(\mathrm{R}^{2}=0.99\right)$ when fit with a log linear curve (Fig. 2$)$. A log linear curve was fit to the log spore quantity to account for the number of primers used in the assay, and the amplicon produced concatenates resulting in greater than an exponential rate of amplification. This curve was used to quantify the L-qLAMP samples collected from the Botany and Plant Pathology Research Farm vineyard. The L-qLAMP spore quantification was significantly lower than the qPCR quantification when daily samples were collected in $2013(P<0.001)$ (Fig. 3A), but the biweekly L-qLAMP and qPCR sample quantification was not significantly different in 2013 ( $P=$ 0.14) (Fig. 3B). The L-qLAMP assay significantly underrepresented spore levels for both the daily collections $(P<0.001)$ (Fig. 4A) and the biweekly collections $(P=0.01)$ (Fig. 4B) compared to the qPCR assay in 2014.

Lab Conducted qLAMP Detection. Utilizing L-qLAMP for detection of E. necator showed similar results to qPCR assay detections in both 2013 and $2014(P<0.001)$ (Table 2). The L-qLAMP assay detection results were 83\% and 70\% accurate in 2013 and 2014, 
225 respectively compared to the qPCR assay detection results. The L-qLAMP assay detection

226 results showed true negative proportions of $89 \%$ and $94 \%$ and true positive proportions of $78 \%$

227

228

229

230

231

232

233

234

235

236

237

238

239

240

241

242

243

244

245

246

247

and 37\% in in 2013 and 2014, respectively. There was an unexplained loss of sensitivity in 2014 sample testing that was extensively examined (see below).

Grower-Conducted qLAMP Assay. The software provided with the mobile LAMP device used auto-adjusting threshold values to account for noise of fluorescence readings which significantly reduced accurate quantification by growers. The G-qLAMP assay for the detection of $E$. necator was not correlated to the qPCR detection results $(P=0.22)$ (Table 2$)$. The GqLAMP detection results showed $82 \%$ accuracy compared to the qPCR assay results, respectively. The G-qLAMP detection results show true negative proportions of $94 \%$, and true positive proportions of $18 \%$ compared to the qPCR detection results.

qLAMP Assay Troubleshooting. Due to loss of sensitivity of the qLAMP assays to $E$. necator observed during assay testing in 2014, extensive troubleshooting was conducted. Primer purification, polymerase used (Bst or OptiGene), master mix distributer, assimilating probe removal, primer and assimilating probe manufacturer, inhibitor removal compounds in the master mix, DNA extraction and clean up, adjustment of reaction temperature, and replacement of reagents and primers were all tested. Primer purification was tested prior to the implementation of the experiment, and during the observed degradation of qLAMP sensitivity with no observable difference between reaction efficiency of HPLC or desalted primers.

Regardless of polymerase used, Bst or ISO-001 (Optigene Ltd, West Sussex, UK), reaction efficiency and sensitivity to $E$. necator DNA was reduced compared to assays conducted prior to implementation of field testing. Different distributers of the Optigene Isothermal Mastermix were also tested to determine if the decreased sensitivity was caused by storage or 
248 shipping errors; however, there was no difference among master mix vendors. It was not possible 249 to test previous lots of the master mix prior to the observed decrease in sensitivity. The assimilating probe was removed and gel electrophoresis was used to compare with and without probe presence, and no difference was observed in amplification. There was also no difference between different primer and probe manufacturers, which also suggests there were no differences in manufacturing process.

The concentrations of inhibitor removal compounds within the master mix were assessed, including PVP 40, EDTA, and BSA concentrations, to determine if inhibitor presence was causing decreased reaction efficiency, and no differences were observed for inhibitor removal compounds. In addition to testing master mix removal of inhibitors, three DNA extraction methods [extractions with $\mathrm{pH} 7.5,10 \mathrm{mM}$ Tris-0.1mM EDTA buffer (Affymetrix, Santa Clara, CA, USA), 2\% polyvinylpyrrolidone (PVP) 40 (Sigma Aldrich, St. Louis, MO, USA) in DEPCtreated water, and PowerSoil ${ }^{\circledR}$ DNA extraction kit (Mo Bio Laboratories, Inc. Carlsbad, CA)] were assessed with separate field collected spore samples. No differences were observed in amplification time or efficiency when testing each side-by-side extraction method.

To test the optimal reaction temperature of the polymerase, temperatures between 60 and $70^{\circ} \mathrm{C}$ were examined to find the optimal reaction temperature. Lower spore quantities (10 spores or less) amplified at $62^{\circ} \mathrm{C}$. A last effort to determine if the effect was due to degradation of reagents of primers during the growing season, all reagents, primers, and probe were replaced; however, the decreased sensitivity to E. necator DNA was still observed. Despite targeting various portions of the reaction and extraction, the cause for loss of qLAMP assay sensitivity remains undetermined. 


\section{Discussion}

A highly sensitive qLAMP assay was successfully developed using a simple DNA extraction method for use by growers or crop consultants to use as a decision aid for timing fungicide applications similar to Thiessen et al. $(2016 ; 2017)$. The qLAMP assay developed for the assay was sensitive to E. necator DNA with one spore amplifying $80 \%(\mathrm{n}=10)$ using the simplified DNA extraction. This sensitivity indicated that the assay should be suitable to detect inoculum (i.e. ascospores) at low concentrations ( $<10$ spores) and aid management decisions. However, the qLAMP assay consistently underrepresented spore quantities later in the growing season compared to the qPCR assay, which may be due to the an increase in the presence of PCR inhibitors (such as pollen, humic acids from soil, spider webs, etc.) found in air samples (Wilson 1997) that may not have been removed by the rapid Chelex DNA extraction. In early DNA extraction testing prior to qLAMP sensitivity loss, the PowerSoil ${ }^{\circledR}$ extracted DNA showed more consistent amplification of field samples than the other extraction methods (Thiessen et al. 2016); however, the PowerSoil ${ }^{\circledR}$ DNA extraction kit requires a larger time commitment and several steps that may not be feasible for in-field DNA extractions. The LAMP assay has been widely described as more tolerant to inhibitors than qPCR (Francois et al. 2011; Kaneko et al. 2007), but it appears that the LAMP assay tolerates different inhibitors than the qPCR assay (Nixon et al. 2014). Additionally, the qLAMP $\mathrm{R}_{\mathrm{T}}$ variance from 1 and 10 spore samples (Fig. 2) was so great that they cannot be distinguished. This variance is likely due to using DNA extractions of each spore concentration as opposed a dilution from higher spore concentration as is typically done (Mahaffee \& Stoll 2016). Because the LAMP assay is not limited by temperature cycles, annealing is reliant on proximity of DNA to the polymerase and primer set (Notomi et al. 2000), and the improved sensitivity with lower annealing temperatures is likely 
294 the result of lower specificity of primers rather than optimal reaction temperature. In reactions

295 with lower quantities of DNA (e.g. 1 and 10 spores), more time may be required for the

296

297

298

299

300

301

302

303

304

305

306

307

308

309

310

311

312

313

314

315

316

polymerase, primers, and target DNA to meet, which may explain the variability of $\mathrm{R}_{\mathrm{T}}$ values of low spore quantities (Fig. 2). The inhibition of the field qLAMP assay and the difficulty of differentiating low spore quantities indicates that the assay currently has more utility as a qualitative inoculum detection tool as opposed to quantitative assessment of inoculum availability.

The G-qLAMP results were significantly different from the qPCR detection results $(P=$ 0.22) (Table 2). This may be due to difficulty in assessing positive detections from the output of the mobile device. The curve smoothing algorithm used by the device application (G-qLAMP) often produced curves that drifted linearly with $\mathrm{R}_{\mathrm{T}}$ values reported even though there was no detectable amplification using gel electrophoresis. Growers conducting the q-LAMP assay were directed to ignore curves that ascended linearly due to curve smoothing; however, this may have caused growers to be overly-conservative in determining positive detections. Additionally, the grower-conducted q-LAMP occurred in 2014 when the loss of q-LAMP sensitivity was observed and there was very low disease.

The L-qLAMP assay detection results were similar to qPCR assay detection results in both 2013 and 2014, but true positive and true negative proportions were variable between years. This variability may be due to the presence of inhibitors. In 2013, the source of stainless steel rod material was changed from previous testing, and significant inhibition of DNA amplification was observed. After troubleshooting various rod cleaning processes and DNA extraction techniques, a hexane soak was added to the steel rod cleaning protocol to remove oils prior to sterilization and 5\% Chelex 100 was used as the extraction buffer. After the hexane wash step addition, the 
317 accuracy of samples was improved to $85 \%$, and the misclassification rate was reduced from $17 \%$

318 to $14 \%$. In addition to inhibitors from the rods, the variability of inhibitors from field collections

319

320

321

322

323

324

325

326

327

328

329

330

may have caused inconsistencies in qLAMP assay detection results compared to the qPCR assay

detection results. Early in the growing season, the weather in the region is characterized by

frequent precipitation events that limit pollen and insect flight. Later in the growing season,

pollen, insects, birds, and soil particulates are abundant in the air, and subsequently on the sampling rods. RNAses, DNAses, humic acids, and other heavy metals may not be removed

when using the chelex DNA extraction (qLAMP template), but are removed during the

Powersoil DNA extraction (qPCR template). The results from the qLAMP had lower true

positive proportions and true negative proportions than that of turbidimetric LAMP previously

developed (Thiessen et al. 2016). These reductions may be due to other factors besides

amplification inhibitors, such as manufacturer differences, degradation of polymerase, inclusion

of probes, or buffering of the qLAMP reaction (Corless et al. 2000; Roux 2009).

Using the qLAMP assay for field detection and quantification of fungal pathogens may

not be as feasible as previously thought due to the random loss of assay sensitivity and potential

inhibition of polymerase activity by environmental contaminants. Redesigning primers was

another potential approach to examining the cause of the reduced sensitivity; however, the

primer set used here was the result of two previous redesigns during development and testing and there was not sufficient heterogeneity in other regions of the ITS. Additionally, the LAMP assay quantification was also affected by numerous inhibitors, such as soil, pollen, or insect debris, found in field collected samples. LAMP is capable of tolerating some inhibitors that affect PCR assays (Francois et al. 2011); however, to determine the extent that LAMP assays are capable of tolerating inhibitors, each potential inhibitor should be tested (Nixon et al. 2014). Other LAMP 
340

341

342

343

344

assays developed have utilized more complex DNA extractions to reduce the effect of inhibitors on amplification for quantitation of DNA (Harper et al. 2010; Kubota et al. 2011; Mori et al. 2004); however, complex DNA extraction techniques are likely to preclude field implementation of LAMP assays and increase assay costs. The observed inconsistency indicates that the developed qLAMP assays might not be robust enough for commercial implementation.

The LAMP assay was developed due to reports of high sensitivity and specificity to target DNA, tolerance of the reaction to the presence of reaction inhibitors, and the potential for use by growers or crop consultants using hand-held LAMP devices such as the BioRanger (Diagenetix, Inc., Hawaii, USA) or the Genie II and III (Optigene Ltd, West Sussex, UK) (Kubota et al. 2011; Kubota et al. 2008; Mori et al. 2004; Mori et al. 2001; Notomi et al. 2000; Temple \& Johnson 2011; Tomlinson et al. 2010); however, field testing of the qLAMP assay for E. necator revealed an unidentifiable degradation of the sensitivity of the assay to the target DNA. The qLAMP assay may still be a useful tool for field inoculum detection, but further analysis of the system is required to determine the specific cause of the degradation of the assay. At the time this research was initiated the LAMP technology was the most advanced for inexpensive field application and thus selected for investigation over other potentially suitable technologies. However, other DNA amplification techniques have since become more accessible for field use (Marx 2015), including qPCR (BioMeme, Inc., Philadelphia, PA, USA) and Recombinase Polymerase Amplification (RPA) (Piepenburg et al. 2006), and HelicaseDependent isothermal DNA Amplification (HDA) (Vincent et al. 2004). These assays require minimal DNA preparation, are capable of real-time data, and may be easily adapted to the air samples used here but require evaluation. There are several reviews that discuss the advantages 
362 and disadvantages of these technologies (Craw \& Balachandran 2012; Gill \& Ghaemi 2008;

363 Mahaffee 2014; Niemz et al. 2011; Yan et al. 2014).

\section{Conclusions}

366

A highly sensitive qLAMP assay was developed using a simple DNA extraction method for use by growers or crop consultants utilizing inoculum detection; however, the qLAMP assay consistently underrepresented spore quantities later in the growing season compared to the qPCR assay. Additionally, the qLAMP assay lost sensitivity to low spore quantities $(<10$ spores $)$ in the 2014 sampling period, and the cause was not determined during the course of this study.

Grower-conducted inoculum monitoring technologies, like the qLAMP assay developed in this study, may provide an inexpensive tool for producers to apply targeted fungicide applications based on inoculum presence and concentration. Given the limitations described herein, more assessment of the qLAMP assay degradation is necessary before utilizing it as a monitoring tool for E. necator inoculum concentrations.

\section{Acknowledgments}

We thank the technical support of Andy Albrecht, Cole Provence, Chris Gorman, and Jim Eynard. We also thank anonymous reviewers for their helpful suggestions to improve the manuscript. We especially thank the numerous vineyard managers that collaborated on the project. The use of trade, firm, or corporation names in this publication is for the information and convenience of the reader. Such use does not constitute an official endorsement or approval by the United States Department of Agriculture or the Agricultural Research Service of any product or service to the exclusion of others that may be suitable. 


\section{References}

Cardoso TC, Ferrari HF, Bregano LC, Silva-Frade C, Rosa ACG, and Andrade AL. 2010. Visual detection of turkey coronavirus RNA in tissues and feces by reverse-transcription loop-mediated isothermal amplification (RTLAMP) with hydroxynaphthol blue dye. Molecular and cellular probes 24:415-417.

Carisse O, Bacon R, and Lefebvre A. 2009a. Grape powdery mildew (Erysiphe necator) risk assessment based on airborne conidium concentration. Crop Prot 28:1036-1044.

Carisse O, Bacon R, Lefebvre A, and Lessard K. 2009b. A degree-day model to initiate fungicide spray programs for management of grape powdery mildew (Erysiphe necator). Canada Journal of Plant Pathology 31:186194.

Carisse O, Tremblay DM, Lévesque CA, Gindro K, Ward P, and Houde A. 2009c. Development of a TaqMan realtime PCR assay for quantification of airborne conidia of Botrytis squamosa and management of Botrytis leaf blight of onion. Phytopathology 99:1273-1280. HTTP://DX.DOI.ORG/10.1094/PHYTO-99-11-1273

Corless CE, Guiver M, Borrow R, Edwards-Jones V, Kaczmarski EB, and Fox AJ. 2000. Contamination and sensitivity issues with a real-time universal 16S rRNA PCR. Journal of clinical microbiology 38:17471752.

Craw P, and Balachandran W. 2012. Isothermal nucleic acid amplification technologies for point-of-care diagnostics: a critical review. Lab on a Chip 12:2469-2486.

Falacy JS, Grove GG, Mahaffee WF, Galloway H, Glawe DA, Larsen RC, and Vandemark GJ. 2007. Detection of Erysiphe necator in air samples using the polymerase chain reaction and species-specific primers. Phytopathology 97:1290-1297.

Fischbach J, Xander NC, Frohme M, and Glökler JF. 2015. Shining a light on LAMP assays - A comparison of LAMP visualization methods including the novel use of berberine. BioTechniques 58:189-194.

Francois P, Tangomo M, Hibbs J, Bonetti E-J, Boehme CC, Notomi T, Perkins MD, and Schrenzel J. 2011. Robustness of a loop-mediated isothermal amplification reaction for diagnostic applications. FEMS Immunology \& Medical Microbiology 62:41-48.

Gadoury DM, and Pearson RC. 1990. Ascocarp dehiscence and ascospore discharge in Uncinula necator. Phytopathology 80:393-401.

Gill P, and Ghaemi A. 2008. Nucleic acid isothermal amplification technologies-a review. Nucleosides, Nucleotides, and Nucleic Acids 27:224-243.

Harper SJ, Ward LI, and Clover GR. 2010. Development of LAMP and real-time PCR methods for the rapid detection of Xylella fastidiosa for quarantine and field applications. Phytopathology 100:1282-1288. 10.1094/PHYTO-06-10-0168

Jenkins DM, Kubota R, Dong J, Li Y, and Higashiguchi D. 2011. Handheld Device for Real-Time, Quantitative, LAMP-Based Detection of Salmonella enterica Using Assimilating Probes. Biosensors and Bioelectronics 30:255-260. 10.1016/j.bios.2011.09.020

Kaneko H, Kawana T, Fukushima E, and Suzutani T. 2007. Tolerance of loop-mediated isothermal amplification to a culture medium and biological substances. Journal of biochemical and biophysical methods 70:499-501.

Kubota R, Alvarez AM, Su WW, and Jenkins DM. 2011. FRET-Based Assimilating Probe for Sequence-Specific Real-Time Monitoring of Loop-Mediated Isothermal Amplification (LAMP). Biological Engineering Transactions 4:81-100.

Kubota R, Vine BG, Alvarez aM, and Jenkins DM. 2008. Detection of Ralstonia solanacearum by loop-mediated isothermal amplification. Phytopathology 98:1045-1051. 10.1094/PHYTO-98-9-1045

Mahaffee WF. 2014. Use of airborne inoculum detection for disease management decisions. Detection and diagnostics of plant pathogens: Springer, 39-54.

Mahaffee WF, and Stoll R. 2016. The ebb and flow of airborne pathogens: monitoring and use in disease management decisions. Phytopathology 106:420-431.

Marx V. 2015. PCR heads into the field. Nature Methods 12:393-397. 10.1038/nmeth.3369

Mori Y, Kitao M, Tomita N, and Notomi T. 2004. Real-time turbidimetry of LAMP reaction for quantifying template DNA. Journal of Biochemical and Biophysical Methods 59:145 - 157. 10.1016/j.jbbm.2003.12.005

Mori Y, Nagamine K, Tomita N, and Notomi T. 2001. Detection of loop-mediated isothermal amplification reaction by turbidity derived from magnesium pyrophosphate formation. Biochemical and biophysical research communications 289:150-154. 10.1006/bbrc.2001.5921

Niemz A, Ferguson TM, and Boyle DS. 2011. Point-of-care nucleic acid testing for infectious diseases. Trends in biotechnology 29:240-250. 
441

442

443

444

445

446

447

448

449

450

451

452

453

454

455

456

457

458

459

460

461

462

463

464

465

466

467

468

469

470

471

472

473

474

475

Nixon G, Garson JA, Grant P, Nastouli E, Foy CA, and Huggett JF. 2014. Comparative study of sensitivity, linearity, and resistance to inhibition of digital and nondigital polymerase chain reaction and loop mediated isothermal amplification assays for quantification of human cytomegalovirus. Analytical chemistry $86: 4387-4394$.

Notomi T, Okayama H, Masubuchi H, Yonekawa T, Watanabe K, Amino N, and Hase T. 2000. Loop-mediated isothermal amplification of DNA. Nucleic acids research 28:E63.

Piepenburg O, Williams CH, Stemple DL, and Armes NA. 2006. DNA detection using recombination proteins. PLoS biology 4:e204.

Rogers SL, Atkins SD, and West JS. 2009. Detection and quantification of airborne inoculum of Sclerotinia sclerotiorum using quantitative PCR. Plant Pathology 58:324-331. 10.1111/j.1365-3059.2008.01945.x

Roux KH. 2009. Optimization and troubleshooting in PCR. Cold Spring Harbor Protocols 2009:pdb. ip66.

Temple TN, and Johnson KB. 2011. Evaluation of Loop-Mediated Isothermal Amplification for Rapid Detection of Erwinia amylovora on Pear and Apple Fruit Flowers. Plant Disease 95:423-430.

Thiessen L, Neill T, and Mahaffee W. 2017. Timing Fungicide Application Intervals Based on Airborne Erysiphe necator Concentrations. Plant Disease:PDIS-12-16-1727-RE.

Thiessen LD, Keune JA, Neill TM, Turechek WW, Grove GG, and Mahaffee WF. 2016. Development of a grower-conducted inoculum detection assay for management of grape powdery mildew. Plant Pathology 65:238-249.

Thomas CS, Gubler WD, and Leavitt G. 1994. Field testing of a powdery mildew disease forecast model on grapes in California. Phytopathology. p 1070 (abstr.).

Tomlinson JA, Barker I, and Boonham N. 2007. Faster, simpler, more-specific methods for improved molecular detection of Phytophthora ramorum in the field. Applied and Environmental Microbiology 73:4040-4047. 10.1128/AEM.00161-07

Tomlinson JA, Dickinson MJ, and Boonham N. 2010. Detection of Botrytis cinerea by loop-mediated isothermal amplification. Letters in applied microbiology 51:650-657. 10.1111/j.1472-765X.2010.02949.x

Vincent M, Xu Y, and Kong H. 2004. Helicase-dependent isothermal DNA amplification. EMBO reports 5:795-800. West JS, Atkins SD, Emberlin J, and Fitt BDL. 2008a. PCR to predict risk of airborne disease. Trends Microbiol 16:380-387.

West JS, Atkins SD, Emberlin J, and Fitt BDL. 2008b. PCR to predict risk of airborne disease. Trends in Microbiology 16:380-387.

Wilson IG. 1997. Inhibition and facilitation of nucleic acid amplification. Applied and Environmental Microbiology 63:3741.

Yan L, Zhou J, Zheng Y, Gamson AS, Roembke BT, Nakayama S, and Sintim HO. 2014. Isothermal amplified detection of DNA and RNA. Molecular BioSystems 10:970-1003. 


\section{Table $\mathbf{1}$ (on next page)}

Primers and probes used for the detection of Erysiphe necator ITS region.

${ }^{\text {a }}$ Primers and probe from qPCR assay and primers from the LAMP assay developed by Thiessen et al. (2016) were used to develop and test the quantitative LAMP assay. ${ }^{\mathrm{b}}$ Primer concentrations in the reaction mix were $2.4 \mu \mathrm{M}$ for FIP and BIP, $0.24 \mu \mathrm{M}$ for F3 and B3, and $0.8 \mu \mathrm{M}$ for Forward Loop primer FAM strand (FL-F) and Quencher strand (Q-strand). Melting temperatures for the primers were between 64 and $99^{\circ} \mathrm{C}$. ${ }^{\mathrm{C}}$ Primer concentrations in the reaction mix were $400 \mathrm{nM}$ for Unc144 Forward, Unc511 Reverse, and the Unc TaqMan ${ }^{\circledR}$ Probe. Melting temperatures for the primers were 59.2 and $59.9^{\circ} \mathrm{C}$, respectively. 
1 TABLE 1. Primers and probes used for the detection of Erysiphe necator ITS region.

\begin{tabular}{lll}
\hline \multicolumn{2}{c}{ Primer/probe $^{\mathrm{a}}$} & \\
\hline qLAMP $^{\mathrm{b}}$ & & Nucleotide Sequence $\left(5^{\prime} \rightarrow 3^{\prime}\right)$ \\
& FIP EN & ACCGCCACTGTCTTTAAGGGCCTTGTGGTGGCTTCGGTG \\
BIP EN & GCGTGGGCTCTACGCGTAGTAGGTTCTGGCTGATCACGAG \\
F3 EN & TCATAACACCCCCCTCAAGCTGCC \\
B3 EN & AACCTGTCAATCCGGATGAC \\
FL-F & FAM-ACGCTGAGGACCCGGATGCGAATGCGGATGCGGATGCCGAAAACTGCGACGAGCCCC \\
Q-Strand & TCGGCATCCGCATCCGCATTCGCATCCGGGTCCTCAGCGT-BHQ \\
qPCR & & \\
& & \\
Unc144 Forward & CCGCCAGAGACCTCATCCAA \\
Unc511 Reverse & TGGCTGATCACGAGCGTCAC \\
Unc TM Probe & 6FAM*-ACGTTGTCATGTAGTCTAA-MGBNFQ
\end{tabular}

2 a Primers and probe from qPCR assay and primers from the LAMP assay developed by Thiessen et al. (2016)

3 were used to develop and test the quantitative LAMP assay.

$4 \quad{ }^{\mathrm{b}}$ Primer concentrations in the reaction mix were $2.4 \mu \mathrm{M}$ for FIP and BIP, $0.24 \mu \mathrm{M}$ for F3 and B3, and 0.8

$5 \mu \mathrm{M}$ for Forward Loop primer FAM strand (FL-F) and Quencher strand (Q-strand). Melting temperatures for

6 the primers were between 64 and $99^{\circ} \mathrm{C}$.

7 c Primer concentrations in the reaction mix were $400 \mathrm{nM}$ for Unc144 Forward, Unc511 Reverse, and the Unc

8 TaqMan ${ }^{\circledR}$ Probe. Melting temperatures for the primers were 59.2 and $59.9^{\circ} \mathrm{C}$, respectively. 


\section{Table 2 (on next page)}

Contingency table representing the lab quantitative LAMP assay and grower quantitative LAMP assay compared to quantitative PCR (qPCR) detection results

Contingency table representing the lab quantitative LAMP assay and grower quantitative LAMP assay compared to quantitative PCR ( $(\mathrm{PPCR}$ ) detection results for the presence of Erysiphe necator sampled from custom made impaction spore samplers from both commercial vineyards and research plots at the Oregon State University Botany and Plant Pathology Research Vineyard. 
1 Table 2. Contingency table representing the lab quantitative LAMP assay and grower

2 quantitative LAMP assay compared to quantitative PCR (qPCR) detection results for the

3 presence of Erysiphe necator sampled from custom made impaction spore samplers from both

4 commercial vineyards and research plots at the Oregon State University Botany and Plant

5 Pathology Research Vineyard.

6

\begin{tabular}{cccccc} 
& & \multicolumn{2}{c}{ qPCR $^{\mathrm{c}}$} & \multirow{2}{*}{$\begin{array}{c}\text { Fisher's Exact Test } \\
\text { (Probability) }^{\mathrm{d}}\end{array}$} \\
\cline { 3 - 4 } & \multirow{2}{*}{2013} & Positive & $146(46 \%)$ & $13(4 \%)$ & $<0.0001^{*}$ \\
Laboratory- & & Negative & $42(13 \%)$ & $115(37 \%)$ & \\
qLAMPa & \multirow{2}{*}{2014} & Positive & $36(16 \%)$ & $8(3 \%)$ & $<0.0001^{*}$ \\
& & Negative & $61(27 \%)$ & $123(54 \%)$ & \\
Grower- & \multirow{2}{*}{2014} & Positive & $2(3 \%)$ & $4(5 \%)$ & $0.22^{*}$ \\
qLAMP & & & & & \\
\hline
\end{tabular}

7 a "Positive" and "Negative" indicate the number of samples for which E. necator DNA was detected and not

8 detected, respectively as tested by L-qLAMP ( $n=316$ in 2013 and $n=228$ in 2014) assays as described in the text.

9 b G-qLAMP ( $n=73$ in 2014) assessed by growers using mobile qLAMP devices (Diagenetix Inc., Honolulu,

$10 \mathrm{HI})$ as described in the text.

$11{ }^{\mathrm{c}} \mathrm{qPCR}$ results based on TaqMan ${ }^{\circledR}$ probe with minor groove binder for detecting E. necator DNA. "Positive"

12 and "Negative" indicate the number of samples for which E. necator DNA was detected and not detected,

13 respectively. qPCR detection data based on quantitative data from (Thiessen et al. 2017).

14 d Fisher's exact test was used to assess the null hypothesis that each LAMP assay was significantly different

15 from the qPCR assay.

$16 *$ significant chi-squared test at $P<0.05$ of qLAMP and qPCR assays. 


\section{Figure 1 (on next page)}

Sensitivity of qLAMP assay to Erysiphe necator as a function of percent amplification (yaxis) and spore $+1 \log _{10}$ concentrations (x-axis).

Each point represents the amplification of 10 separate extractions created from different $E$. necator conidia dilution series ( $10^{2}, 10^{3}$, and $10^{4}$ conidia concentrations), 1 and 10 conidia eyelash transferred spore rods, and conidia-free spore rods $(n=10)$. 


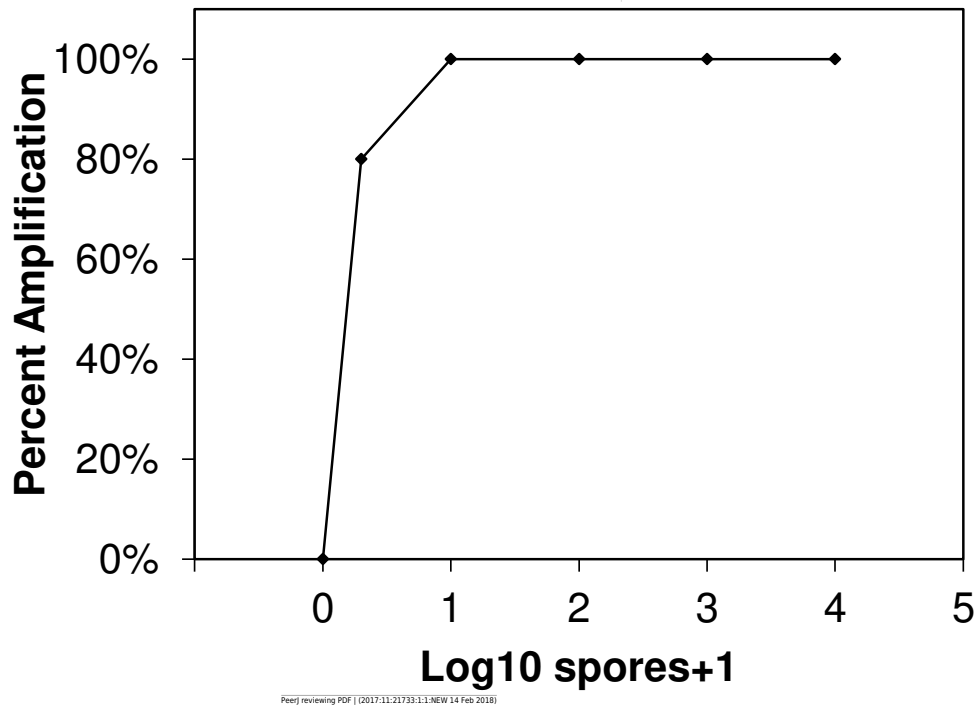




\section{Figure 2 (on next page)}

qLAMP standard curve developed from 6 separate Erysiphe necator spore dilution series comparing the spore $+1 \log _{10}$ quantity to the reaction time-threshold $\left(R_{T}\right)$ value (minutes).

The average $R_{T}$ value was used to determine the spore quantities of unknown samples. 
Figure 3 (on next page)

Erysiphe necator spore enumeration in 2013.

Erysiphe necator spore enumeration determined by qLAMP (gray diamond) and qPCR (black square) assays collected daily (A) and biweekly (B) from the Botany and Plant Pathology Research Farm vineyard (Corvallis, OR) during the 2013 growing season. The qLAMP spore quantification was significantly lower than the qPCR daily samples $(P<0.001)$, but the biweekly qLAMP and qPCR sample quantification was not significantly different $(P=0.14)$. 
Figure 4 (on next page)

Erysiphe necator spore enumeration in 2014

Erysiphe necator spore enumeration determined by qLAMP (gray diamond) and qPCR (black square) assays collected daily (A) and biweekly (B) from the Botany and Plant Pathology Research Farm vineyard (Corvallis, OR) during the 2014 growing season. The qLAMP assay significantly underrepresented spore levels for both the daily collections $(P<0.001)$ and the biweekly collections $(P=0.01)$ compared to the qPCR assay. 
